\title{
Fusing Speed and Phase Information for Vascular Segmentation in Phase Contrast MR Angiograms
}

\author{
Albert C. S. Chung ${ }^{1}$, J. Alison Noble ${ }^{1}$ and Paul Summers ${ }^{2}$ \\ ${ }^{1}$ Department of Engineering Science, Oxford University, Oxford, United Kingdom. \\ 2 Department of Clinical Neuroscience, King's College London, United Kingdom. \\ Email: \{albert, noble\}@robots.ox.ac.uk, p.summers@iop.kcl.ac.uk
}

\begin{abstract}
This paper presents a statistical approach to aggregating speed and phase (directional) information for vascular segmentation in phase contrast magnetic resonance angiograms (PC-MRA), and proposes a Maxwell-Gaussian finite mixture distribution to model the background noise distribution. In this paper, we extend our previous work [6] to the segmentation of phase-difference PC-MRA speed images. We demonstrate that, rather than relying on speed information alone, as done by others $[12,14,15]$, including phase information as $a$ priori knowledge in a Markov random field (MRF) model can improve the quality of segmentation, especially the region within an aneurysm where there is a heterogeneous intensity pattern and significant vascular signal loss. Mixture model parameters are estimated by the Expectation-Maximization (EM) algorithm [3]. In addition, it is shown that a Maxwell-Gaussian finite mixture distribution models the background noise more accurately than a Maxwell distribution and exhibits a better fit to clinical data.
\end{abstract}

Keywords: Medical image processing, Statistical segmentation and Medical information fusion.

\section{Introduction}

Medical diagnosis of vascular diseases is commonly performed on the basis of MRA speed images, which assign high intensity to the moving blood and CSF. The higher the velocity, the higher the image intensity level assigned to it. While most of the arterial anatomy can be shown clearly in the speed images, objects such as intracranial aneurysms containing low and complex flow [17] are poorly represented in the images. The presence of an aneurysm causes significant vascular signal loss in the MRA speed image with some intensity levels approximately equal to those of background tissue, thereby, producing a heterogeneous intensity pattern within the aneurysm. This is problematic in vascular segmentation.

Phase contrast magnetic resonance angiography (PC-MRA) gives not only speed information, but also encodes directional information, which is represented by three separate phase images. Each phase image represents a directional component of the flow. Typical methods for segmentation of MRA images include geodesic active contours implemented using level set methods [14], multiscale-based tubular structure detection [12] and topologically adaptable surfaces [15]. Rather than using just speed information, the directional flow pattern may give additional clues for segmentation. To the best of our knowledge, there is only one related work [19] using both speed and phase images, which uses a multi-resolution, model-based approach to extracting and visualizing vascular flow features.

The method we propose here draws on the fact that the flow pattern in the vasculature is locally coherent. In other words, if blood is flowing in a direction $v$, 
neighbouring voxels should have a high probability of exhibiting flow in the same direction $\boldsymbol{v}$. Local phase coherence measures can be derived to estimate the degree of coherence amongst neighbouring voxels. In this paper, we present a statistical approach, which incorporates the local phase coherence as a priori knowledge in a Markov random field (MRF) model to improve the quality of vascular segmentation. Related works can be found in the literature of MRF-based segmentation of conventional MR images, rather than MRA, including mean field approximation [11], ICM [9] and MRF-EM [20].

Moreover, in this paper, we derive the background noise statistical model based on knowledge of the image formation process and use it to derive update equations for Expectation-Maximization (EM) based parameter estimation. In addition, it is shown that the proposed Maxwell-Gaussian finite mixture distribution noise model represents the noise more accurately than a Maxwell distribution used in prior work [1] and shows a better fit to clinical data. It should be noted that a Maxwell distribution describes the probability distribution of the modulus of three Gaussians with zero-mean and equal variance, and the widely known Rayleigh distribution describes the probability distribution of the modulus of two Gaussians with zero-mean and equal variance.

\section{Statistical Analysis of Noise and Vascular Signals}

The phase angle of a complex-valued MR signal $\mathrm{S}_{1}$ is defined as $\phi_{1}$ and computed by $\arg \left(\mathrm{S}_{1}\right)=\tan ^{-1}\left(\operatorname{Im}\left\{\mathrm{S}_{1}\right\} / \operatorname{Re}\left\{\mathrm{S}_{1}\right\}\right)$, where $\operatorname{Re}\left\{\mathrm{S}_{1}\right\}$ and $\operatorname{Im}\left\{\mathrm{S}_{1}\right\}$ denote real and imaginary components of the signal respectively, as shown in Figure 1. It is assumed that both real and imaginary components are statistically independent between the two quadrature channels and corrupted by zero-mean Gaussian noise with equal variance $\sigma^{2}$. The probability density function (PDF) of $\phi_{1}$ is given by [13],

$\mathrm{f}_{\phi_{1}}\left(\phi_{1}\right)=\frac{\exp \left(-\alpha^{2}\right)}{2 \pi}\left\{1+\sqrt{\pi} \alpha \cdot \cos \left(\phi_{1}-\overline{\phi_{1}}\right) \cdot \exp \left(\alpha^{2} \cdot \cos ^{2}\left(\phi_{1}-\overline{\phi_{1}}\right)\right) \cdot\left[1+\operatorname{erf}\left(\alpha \cdot \cos \left(\phi_{1}-\overline{\phi_{1}}\right)\right)\right]\right\}$,

where $\phi_{1} \in[-\pi, \pi), \alpha=\mathrm{A} / \sqrt{2} \sigma, \operatorname{erf}(\mathrm{x})=(2 / \sqrt{\pi}) \cdot \int_{0}^{\mathrm{x}} \mathrm{e}^{-\mathrm{w}^{2}} \mathrm{dw}$ is the error function, $\overline{\phi_{1}}$ is the mean phase and $A$ is the deterministic speed of signal $S_{1} . f_{\phi_{1}}=0$ for $\phi_{1} \notin[-\pi, \pi)$. The PDFs at different Signal-to-Noise ratios ( $\mathrm{SNR}=\mathrm{A} / \sigma=0,1,3$ and 6 ) are plotted in Figure 2. It is noted that the PDF becomes uniformly distributed when the SNR is extremely low, and tends to a Gaussian distribution when the SNR is sufficiently high. To study the goodness-of-fit of a Gaussian approximation at various levels, the PDF was fitted by a Gaussian distribution, where mean and variance were estimated by $\mu=\sum \phi_{1} \mathrm{f}\left(\phi_{1}\right)$ and $\sigma^{2}=\sum\left(\phi_{1}-\mu\right)^{2} \mathrm{f}\left(\phi_{1}\right)$ respectively. The absolute difference errors between the PDF and the fitted Gaussian distribution are plotted in Figure 3 at various SNR levels. It is observed that when the SNR is larger than 3, a Gaussian gives a fairly good approximation with absolute difference error less than $8 \%$ of the PDF. In this paper, we assume that the SNR is larger than or equal to 3 and the PDF is approximated by a Gaussian. In our experience, this assumption is valid in clinical practice. For a SNR less than 3, a Gaussian approximation is not sufficient, and special attention must be paid to deal with the long and uniform tails at both sides of the PDF, which forms part of our current work [18]. 
For each velocity component, a phase shift $\Delta \phi$ is produced by the angular difference of the two signal phases [16], i.e. $\Delta \phi=\phi_{2}-\phi_{1}=\arg \left(\mathrm{S}_{2}\right)-\arg \left(\mathrm{S}_{1}\right)$, as shown in Figure 1. The two complex-valued signals, $\mathrm{S}_{2}$ and $\mathrm{S}_{1}$, are acquired along a specific scanning direction by applying two opposite bipolar gradients. The resulting net phase shift $\Delta \phi$ is directly proportional to the flow rate at this voxels.

As mentioned earlier, the PDFs of $\phi_{1}$ and $\phi_{2}$ are assumed Gaussian. Since the difference between two Gaussians is also a Gaussian, the PDF of $\Delta \phi, f_{\Delta \phi}$, follows a zero-mean Gaussian distribution with variance $2 \sigma^{2}$. Figure 4 and Figure 5 show respectively a computer simulated phase image with low SNR and its histogram fitted by a Gaussian distribution.

A MRA speed image is reconstructed on a voxel-by-voxel basis by taking the modulus of the three corresponding phase shift values, $\Delta \phi_{\mathrm{x}}, \Delta \phi_{\mathrm{y}}$ and $\Delta \phi_{\mathrm{z}}$, i.e. $\mathrm{i} \propto \sqrt{\Delta \phi_{\mathrm{x}}^{2}+\Delta \phi_{\mathrm{y}}^{2}+\Delta \phi_{\mathrm{z}}^{2}}$, where $\mathrm{i}$ is the image intensity. Since the phase shifts are flow sensitized along the three directional components, $\mathrm{x}, \mathrm{y}$ and $\mathrm{z}$, and directly proportional to the flow rate, the reconstructed image is called speed image. The distribution described by the modulus of three independent zero-mean Gaussians is a Maxwell distribution [1]. Hence, for the background noise, the noise PDF is given by a Maxwell distribution $f_{M}(i)=\left(2 \cdot i^{2} / \sigma_{M}^{3} \sqrt{2 \pi}\right) \cdot \exp \left(-i^{2} / 2 \sigma_{M}^{2}\right)$, where $\sigma_{M}=\sqrt{2} \sigma$ and $\mathrm{i} \geq 0$. Figure 7 and Figure 8 show respectively a computer simulated speed image with low SNR and its histogram fitted by a Maxwell distribution using the relationship $\sigma_{M}=I_{\text {peak }} / \sqrt{2}$, where $I_{\text {peak }}$ is the intensity value at which the histogram achieves its maximum, i.e. $\mathrm{df}_{\mathrm{M}}(\mathrm{i}) / \mathrm{di}=0$ at $\mathrm{i}=\mathrm{I}_{\text {peak }}$. It is observed that the Maxwell distribution fits well in the low intensity region, but not in the intensity region indicated by the arrow in Figure 8. The reason is that both tails of the histogram are not perfectly fitted by a Gaussian distribution (indicated by the arrows in Figure 5). The absolute difference between them is shown in Figure 6 (with a smaller scale on the vertical axis than Figure 5), and reveals one positive residual distribution located at each side. We assume that these residual distributions are non-zero mean Gaussian. Therefore, for each encoding direction, the PDF $f_{\Delta \phi}$ consists of a zero-mean Gaussian (located at the centre) and two non-zero mean Gaussian distributions (located at each side). After the modulus operation, as mentioned earlier, a Maxwell distribution is formed by the modulus of the three zero-mean Gaussian distributions, whereas, the modulus of the residual non-zero mean Gaussian distributions gives a Gaussian distribution [1]. Hence, the noise PDF consists of a linear mixture of a Maxwell and a Gaussian distribution with mean $\mu_{\mathrm{G}}$ and variance $\sigma_{\mathrm{G}}^{2}$.

As shown in Figure 9, using the EM algorithm [3], a Maxwell and Gaussian mixture noise model achieves a better fit to the given histogram of a PC-MRA speed image with low SNR. To model the vascular signal with high SNR, we apply the results of our previous work [6] and assume that the signal is uniformly distributed. Thus, we conclude that the overall PDF f(i) of a speed image can be modelled as a Maxwell-Gaussian and uniform finite mixture distribution, which is given by 
$\mathrm{f}(\mathrm{i})=\underbrace{\mathrm{w}_{\mathrm{M}} \mathrm{f}_{\mathrm{M}}(\mathrm{i})+\mathrm{w}_{\mathrm{G}} \mathrm{f}_{\mathrm{G}}(\mathrm{i})}_{\text {Background Noise }}+\underbrace{\mathrm{w}_{\mathrm{U}} \mathrm{f}_{\mathrm{U}}(\mathrm{i})}_{\text {Vascular Signal }}$, where $\mathrm{w}_{\mathrm{M}}, \mathrm{w}_{\mathrm{G}}$ and $\mathrm{w}_{\mathrm{U}}$ are weights (or prior probabilities) assigned to the Maxwell, Gaussian and uniform distributions respectively and $\mathrm{w}_{\mathrm{M}}+\mathrm{w}_{\mathrm{G}}+\mathrm{w}_{\mathrm{U}}=1$.

\begin{tabular}{|c|c|c|c|}
\hline \\
Figure 1: Signal phase \\
Shift $\Delta \phi$.
\end{tabular}

\section{Segmentation Algorithm}

The proposed mixture model has six parameters: $\mathrm{w}_{\mathrm{M}}, \mathrm{w}_{\mathrm{G}}, \mathrm{w}_{\mathrm{U}}, \sigma_{\mathrm{M}}^{2}, \mu_{\mathrm{G}}$ and $\sigma_{\mathrm{G}}^{2}$, which need to be estimated. The modified EM algorithm can be used to estimate the parameters by maximizing the log-likelihood of the mixture distribution in each iteration [3]. Let $\mathrm{N}$ be the total number of voxels and $\mathrm{h}(\mathrm{i})$ be the observed histogram distribution. The update equations can be derived and are listed in Table 1.

In our implementation, the initial estimates were found empirically, and convergence was generally reached after about 5 iterations. A typical result is shown in Figure 21. For comparison, a Maxwell and uniform mixture model has been fitted using the EM algorithm [3] in Figure 20, which reveals that the Maxwell model provides a poorer fit than the Maxwell-Gaussian mixture model for background noise. It is also worth noting that, together with the imperfection of the Maxwell noise model, ghosting artifacts and partial volume effect also contribute to the poorer fit of the Maxwell noise model in the clinical data. 


\begin{tabular}{|l|l|}
\hline $\begin{array}{l}\text { Maxwell: } \\
\text { (M) }\end{array}$ & $\mathrm{w}_{\mathrm{M}}^{\mathrm{k}+1}=\frac{1}{\mathrm{~N}} \cdot \sum_{\mathrm{i}} \mathrm{h}(\mathrm{i}) \cdot \mathrm{P}^{\mathrm{k}}(\mathrm{M} \mid \mathrm{i})$ and $\left(\sigma_{\mathrm{M}}^{2}\right)^{\mathrm{k}+1}=\frac{\sum_{\mathrm{i}} \mathrm{h}(\mathrm{i}) \cdot \mathrm{P}^{\mathrm{k}}(\mathrm{M} \mid \mathrm{i}) \cdot \mathrm{i}^{2}}{3 \cdot \sum_{\mathrm{i}} \mathrm{h}(\mathrm{i}) \cdot \mathrm{P}^{\mathrm{k}}(\mathrm{M} \mid \mathrm{i})}$, \\
& where $\mathrm{P}^{\mathrm{k}}(\mathrm{M} \mid \mathrm{i})=\mathrm{w}_{\mathrm{M}}^{\mathrm{k}} \mathrm{f}_{\mathrm{M}}^{\mathrm{k}}(\mathrm{i}) / \mathrm{f}^{\mathrm{k}}(\mathrm{i}) \cdot$ \\
$\begin{array}{l}\text { Gaussian: } \\
(\mathrm{G})\end{array}$ & $\mathrm{w}_{\mathrm{G}}^{\mathrm{k}+1}=\frac{1}{\mathrm{~N}} \cdot \sum_{\mathrm{i}} \mathrm{h}(\mathrm{i}) \cdot \mathrm{P}^{\mathrm{k}}(\mathrm{G} \mid \mathrm{i}), \mu_{\mathrm{G}}^{\mathrm{k}+1}=\frac{\sum_{\mathrm{i}} \mathrm{h}(\mathrm{i}) \cdot \mathrm{P}^{\mathrm{k}}(\mathrm{G} \mid \mathrm{i}) \cdot \mathrm{i}}{\sum_{\mathrm{i}} \mathrm{h}(\mathrm{i}) \cdot \mathrm{P}^{\mathrm{k}}(\mathrm{G} \mid \mathrm{i})}$ and \\
& $\left(\sigma_{\mathrm{G}}^{2}\right)^{\mathrm{k}+1}=\frac{\sum_{\mathrm{i}} \mathrm{h}(\mathrm{i}) \cdot \mathrm{P}^{\mathrm{k}}(\mathrm{G} \mid \mathrm{i}) \cdot\left(\mathrm{i}-\mu_{\mathrm{G}}^{\mathrm{k}}\right)^{2}}{\sum_{\mathrm{i}} \mathrm{h}(\mathrm{i}) \cdot \mathrm{P}^{\mathrm{k}}(\mathrm{G} \mid \mathrm{i})}$, where $\mathrm{P}^{\mathrm{k}}(\mathrm{G} \mid \mathrm{i})=\mathrm{w}_{\mathrm{G}}^{\mathrm{k}} \mathrm{f}_{\mathrm{G}}^{\mathrm{k}}(\mathrm{i}) / \mathrm{f}^{\mathrm{k}}(\mathrm{i})$. \\
\hline $\begin{array}{l}\text { Uniform: } \\
\text { (U) }\end{array}$ & $\mathrm{w}_{\mathrm{U}}^{\mathrm{k}+1}=\frac{1}{\mathrm{~N}} \cdot \sum_{\mathrm{i}} \mathrm{h}(\mathrm{i}) \cdot \mathrm{P}^{\mathrm{k}}(\mathrm{U} \mid \mathrm{i})$, \\
\hline \multicolumn{2}{|c|}{ wable 1: Update equations and posterior probabilities at $\mathrm{k}^{\mathrm{th}}$ iteration for each distribution. } \\
\hline
\end{tabular}

\subsection{Segmentation Based on Speed Information}

Given an estimated mixture model, a PC-MRA speed image can be segmented statistically on the basis of the MAP (Maximum-A-Posteriori) criterion. Using MAP, assuming the weights (or prior probabilities) remain constant, a voxel $\mathrm{x}_{\mathrm{s}} \in\{\mathrm{v}, \mathrm{b}\}$, where $\mathrm{v}$ and $\mathrm{b}$ denote vessel and background respectively, is classified as a vessel voxel when the vessel probability $\mathrm{w}_{\mathrm{U}} \mathrm{f}_{\mathrm{U}}(\mathrm{i})$ is greater than the background noise probability $\mathrm{w}_{M} \mathrm{f}_{\mathrm{M}}(\mathrm{i})+\mathrm{w}_{\mathrm{G}} \mathrm{f}_{\mathrm{G}}(\mathrm{i})$. Therefore, a threshold can be found by seeking the intersection of the background and vessel probability distributions. As indicated by the arrows in Figure 20 and Figure 21, the threshold found by using MaxwellGaussian noise model tends to more correct and is usually higher than that found by using Maxwell noise model. Figure 22 and Figure 23 show a speed image (data provided by the Department of Clinical Neurosciences, King's College London) and a binary-segmented image produced using Maxwell-Gaussian noise model and MAP classification method. The aneurysm is indicated by an arrow in the figure. It is noted that the resulting segmentation is adversely affected by the significant signal loss inside an aneurysm located at the middle. The same happens in Figure 25 and Figure 26. We will discuss how to overcome this problem in the next subsection.

\subsection{Integration of Speed and Phase Information}

Note that the flow pattern in the aneurysm appears to show (a) a vortex centred at the singular point, at which the velocity becomes null, and (b) a locally linear (coherent) motion around the neighbourhood of the singular point, which can also be regarded as a deformed circular flow. This is consistent with the results of clinical flow studies [8] and simulations of computational fluid dynamics [5]. The local linear motion exists not only inside the aneurysm, but also in most of the vasculature having laminar flow pattern. We now present a method for the quantification of these local linear motions.

Let $\mathrm{V}=\left\{\mathrm{v}_{1}, \ldots, \mathrm{v}_{\mathrm{N}}\right\}$ be a velocity map, where $\mathrm{N}$ is the total number of voxels and the three orthogonal velocity components of a voxel $\mathrm{x}_{\mathrm{s}}$ are assigned as the phase shifts in the corresponding phase image, i.e. for $\mathrm{v}_{\mathrm{s}}=\left(\mathrm{v}_{\mathrm{s}}^{\mathrm{x}}, \mathrm{v}_{\mathrm{s}}^{\mathrm{y}}, \mathrm{v}_{\mathrm{s}}^{\mathrm{z}}\right), \mathrm{v}_{\mathrm{s}}^{\mathrm{x}}=\Delta \phi_{\mathrm{x}}$, 
$\mathrm{v}_{\mathrm{s}}^{\mathrm{y}}=\Delta \phi_{\mathrm{y}}$ and $\mathrm{v}_{\mathrm{s}}^{\mathrm{z}}=\Delta \phi_{\mathrm{z}}$. We define a phase feature $\mathrm{P}$ as the cosine of the angle between two velocities, $\mathrm{v}_{\mathrm{s}}$ and $\mathrm{v}_{\mathrm{t}}$, of the neighbouring voxels, $\mathrm{x}_{\mathrm{s}}$ and $\mathrm{x}_{\mathrm{t}}$, where $\mathrm{s}, \mathrm{t} \in[1, \ldots, \mathrm{N}]$. The phase feature $\mathrm{P}$ is given by a dot product of the two normalized velocities, $\mathrm{P}\left(\mathrm{v}_{\mathrm{s}}, \mathrm{v}_{\mathrm{t}}\right)=\mathrm{v}_{\mathrm{s}} \cdot \mathrm{v}_{\mathrm{t}} /\left\|\mathrm{v}_{\mathrm{s}}\right\|\left\|\mathrm{v}_{\mathrm{t}}\right\|$, where $\mathrm{P} \in[-1,1]$. To quantify the local linear motion around a voxels $\mathrm{x}_{\mathrm{s}}$, we measure its local phase coherence (LPC) by applying a $3 \times 3$ voxel mask to the velocity map $V$ at the voxel within slice. LPC is evaluated by considering the locally coherent motion of its 8 neighbouring voxels, which are numbered in Figure 10, and is defined as a circular addition of the phase features around $\mathrm{x}_{\mathrm{s}}$, i.e.

$$
\operatorname{LPC}\left(\mathrm{x}_{\mathrm{s}}\right)=\mathrm{P}\left(\mathrm{v}_{1}, \mathrm{v}_{2}\right)+\mathrm{P}\left(\mathrm{v}_{2}, \mathrm{v}_{3}\right)+\cdots+\mathrm{P}\left(\mathrm{v}_{8}, \mathrm{v}_{1}\right),
$$

where $\operatorname{LPC}\left(\mathrm{x}_{\mathrm{s}}\right) \in[-8,8]$. In other words, LPC is a circular addition of dot products of the 8 adjacent normalized velocity pairs. Figure 12 shows a LPC map for the speed image of Figure 22, in which the image intensity value is directly proportional to the strength of LPC. It is observed that the voxels inside the aneurysm and vessels exhibit high LPC and form a piece-wise homogeneous region, whereas the non-vessel voxels have relatively low and random LPC.

\begin{tabular}{|c|c|c|}
\hline $\mathrm{v}_{1}$ & $\mathrm{v}_{2}$ & $\mathrm{v}_{3}$ \\
\hline $\mathrm{v}_{8}$ & $\mathrm{x}_{\mathrm{s}}$ & $\mathrm{v}_{4}$ \\
\hline $\mathrm{v}_{7}$ & $\mathrm{v}_{6}$ & $\mathrm{v}_{5}$ \\
\hline
\end{tabular}

Figure 10: The 8 neighbouring velocities of voxel $\mathrm{x}_{\mathrm{S}}$.

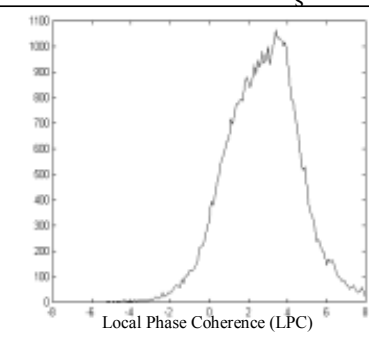

Figure 13: Histogram of the local phase coherence (LPC) map.

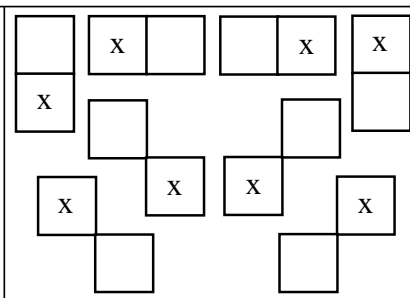

Figure 11: The 8 two-site cliques. $3 \times 3$ mask center is labelled as $\mathrm{x}$

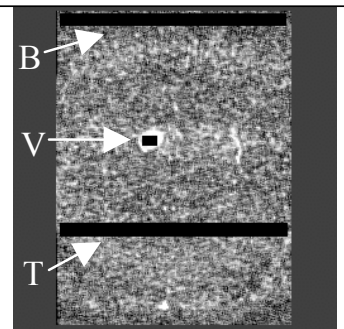

Figure 14: Regions of interest (Black boxes). B - Background, $\mathrm{V}-$ Vessel and T - Brian Tissue

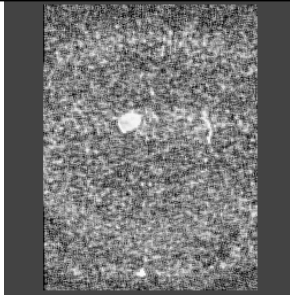

Figure 12: Local phase coherence (LPC) map.

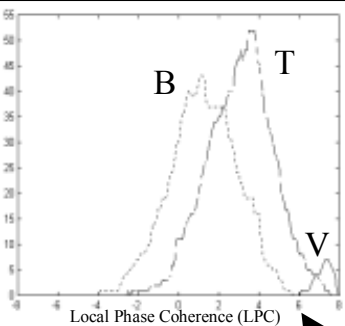
Figure 15: Individual histograms of ROIs.

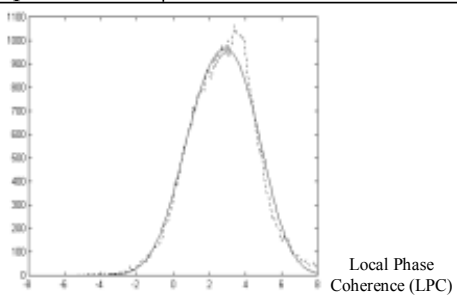

Figure 16: Histogram of LPC map (Dotted) and fitting of twoGaussian model (Solid).

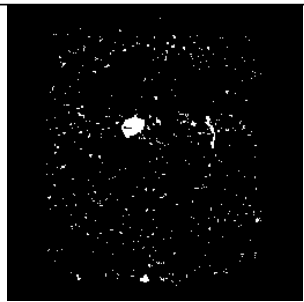

Figure 17: Coherent voxels. 
We now present an automatic threshold determination method for classification of coherent and non-coherent voxels. A histogram of the LPC map is plotted in Figure 13. It shows that the histogram is right shifted and skewed. In fact, the LPC histogram is constituted by three classes: background with low LPC, brain tissue with slightly high LPC, and vessel with extremely high LPC, which are shown in Figure 14 and Figure 15. Background (B) and brain tissue (T) histograms overlap heavily because of the non-stationary, but slightly coherent, motion of the non-vessel brain tissue. In contrast, the vessel (V) histogram is separated clearly from the background (B) histogram. The point of separation - the desired threshold - is indicated by an arrow in Figure 15. We model the background region and nonbackground regions with two separate Gaussian distributions. It is worth noting that the theoretical modelling of the LPC histogram is extremely difficult because of the high correlation factor among the velocity random variables, and normalization and dot product operations of the correlated variables in Equation 1. A Gaussian distribution is employed to model the non-background region including both $\mathrm{T}$ and $\mathrm{V}$ histograms. This is because the $\mathrm{V}$ histogram overlaps heavily with the $\mathrm{T}$ histogram, and occupies only a small portion $(1 \%-4 \%)$ of the LPC histogram. Moreover, as will be discussed below, estimation for the $\mathrm{B}$ histogram is far more important than that for the $\mathrm{T}$ and $\mathrm{V}$ histograms.

We use the EM algorithm [3] to fit the LPC histogram by a mixture of the two Gaussian distributions, as shown in Figure 16. Note that the two estimated Gaussians merge together and form a smooth curve because the means of the two Gaussians are close to each other (in this case, 1.6 for background and 3.7 for nonbackground) and variances are relatively large and roughly the same (in this case, 2.9 for background and 2.2 for non-background). We define the mean and variance of the background distribution as $\mu_{\mathrm{B}}$ and $\sigma_{\mathrm{B}}^{2}$ respectively, and use $\mu_{\mathrm{B}}+3 \cdot \sigma_{\mathrm{B}}$ as a background threshold, which is a variant of the background thresholding approach [4]. A voxel with LPC above the background threshold is labelled as a coherent voxel. Otherwise, it is labelled as a non-coherent voxel. As shown in Figure 17, the coherent voxels form a number of vessel 'clusters', though there are some randomly distributed voxels due to random coherent noise, small coherent motion of the nonvessel tissue during scanning and ghosting artifacts. These 'outliers' can be avoided in the segmentation process by checking their intensity values in the speed image and their interactions with the neighbouring voxels.

A Markov random field framework is used to model the LPC piece-wise homogeneity inside the vasculature. A second-order neighbouring system [7] is used, in which we define 8 two-site cliques, $\mathrm{c}=\{1, \ldots, 8\}$, for each voxel $\mathrm{x}_{\mathrm{s}}$, as shown in Figure 11. This neighbouring system describes the immediate interactions of a voxel with its 8 adjacent in slice voxels. These interactions among the voxels are measured by a clique energy function, which encourages LPC homogeneity and discourages the 'outlier' voxels far away from the vasculature. As such, the clique energy function is defined as

$$
E_{c}\left(x_{s}, x_{i}\right)= \begin{cases}\beta_{v}, & \text { if } x_{s}=x_{i}=v, \text { and } x_{s} \& x_{i} \text { are coherent. } \\ \beta_{b}, & \text { otherwise. }\end{cases}
$$

In this paper, we set $\beta_{\mathrm{v}}$ and $\beta_{\mathrm{b}}$ as 2 and 1 respectively. As such, $\beta_{\mathrm{b}}$ denotes the number of non-coherent or non-vessel neighbouring voxels. In contrast, $\beta_{\mathrm{v}}$ denotes 
twice the number of coherent, vessel voxels in order to favour the coherent, vessel neighbouring voxels. In Section 3.1, the prior probability is assumed constant throughout the segmentation process. Now, the total energy function $\mathrm{U}\left(\mathrm{x}_{\mathrm{s}}\right)$ and prior probability $\mathrm{P}\left(\mathrm{x}_{\mathrm{s}}\right)$ of voxel $\mathrm{x}_{\mathrm{s}}$ are defined as $\mathrm{U}\left(\mathrm{x}_{\mathrm{s}}\right)=\sum_{\mathrm{c}=1}^{8} \sum_{\mathrm{i} \in \mathrm{c}} \mathrm{E}_{\mathrm{c}}\left(\mathrm{x}_{\mathrm{s}}, \mathrm{x}_{\mathrm{i}}\right)$, and $\mathrm{P}\left(\mathrm{x}_{\mathrm{s}}\right)=\mathrm{Z}^{-1} \cdot \exp \left\{\mathrm{U}\left(\mathrm{x}_{\mathrm{s}}\right)\right\}$ respectively, where $\left.\mathrm{Z}=\sum_{\mathrm{x}_{\mathrm{s}} \in[\mathrm{v}, \mathrm{b}}\right] \exp \left\{\mathrm{U}\left(\mathrm{x}_{\mathrm{s}}\right)\right\}$ is a normalization constant. It should be noted that the Markov prior probability is directly proportional to the number of adjacent, and coherent, vessel voxels. Intuitively, the larger the number of adjacent and coherent, vessel voxels around the voxel $\mathrm{x}_{\mathrm{s}}$, the higher the vessel probability $\mathrm{P}\left(\mathrm{x}_{\mathrm{S}}=\mathrm{v}\right)$.

Let $X=\left\{x_{1}, \ldots, x_{N}\right\}$, be the true-segmented image, where $x_{s} \in[v, b]$. Let also $I=\left\{i_{1}, \ldots i_{N}\right\}$ be the observed speed image, where $i_{s} \in\left[0 \ldots I_{\max }\right]$. The Iterated Conditional Modes (ICM) [2] algorithm can be employed to maximize the probability of estimating the true-segmented binary image given the observed image, i.e. $\mathrm{P}(\mathrm{X} \mid \mathrm{I})$. Using the parameters estimated by the modified EM algorithm in the previous section, the vessel likelihood $\mathrm{P}\left(\mathrm{i}_{\mathrm{s}} \mid \mathrm{x}_{\mathrm{s}}=\mathrm{v}\right)$ and background likelihood $\mathrm{P}\left(\mathrm{i}_{\mathrm{s}} \mid \mathrm{x}_{\mathrm{s}}=\mathrm{b}\right)$ are defined as $\mathrm{f}_{\mathrm{U}}\left(\mathrm{i}_{\mathrm{s}}\right)$ and $\left[\mathrm{w}_{\mathrm{M}} \mathrm{f}_{\mathrm{M}}\left(\mathrm{i}_{\mathrm{s}}\right)+\mathrm{w}_{\mathrm{G}} \mathrm{f}_{\mathrm{G}}\left(\mathrm{i}_{\mathrm{s}}\right)\right] /\left(\mathrm{w}_{\mathrm{M}}+\mathrm{w}_{\mathrm{G}}\right)$ respectively. The true segmentation $\mathrm{X}$ is initialized by the segmentation results based on speed information, as shown in Figure 23. The ICM algorithm estimates a voxel class by using MAP method, $\mathrm{x}_{\mathrm{s}}=\arg \max _{\mathrm{x}_{\mathrm{s}} \in[\mathrm{v}, \mathrm{b}]} \mathrm{P}\left(\mathrm{x}_{\mathrm{s}} \mid \mathrm{i}_{\mathrm{s}}\right)$, to maximize the posterior probability $\mathrm{P}\left(\mathrm{x}_{\mathrm{s}} \mid \mathrm{i}_{\mathrm{s}}\right) \propto \mathrm{P}\left(\mathrm{x}_{\mathrm{s}}\right) \cdot \mathrm{P}\left(\mathrm{i}_{\mathrm{s}} \mid \mathrm{x}_{\mathrm{s}}\right)$ at each voxel iteratively until convergence is reached. The convergent rate is usually around 5 iterations.

\section{Results}

Intracranial scans (PC-MRA) of two patients were performed using a 1.5T GE MR scanner at the Department of Clinical Neurosciences, King's College London. The volume size was $256 \times 256 \times 28$ voxels and voxel size $0.8 \mathrm{~mm} \times 0.8 \mathrm{~mm} \times 1 \mathrm{~mm}$. The Maxwell noise model was compared with the proposed Maxwell-Gaussian noise model by computing the absolute difference error for all slices in the two scans. Results are shown in Figure 18 and Figure 19. This shows that Maxwell-Gaussian model is better than the Maxwell model for describing the background noise distribution in PC-MRA speed images by an average of $9 \%$.

The segmentation algorithm was applied to all slices. Typical segmentation results are shown in Figure 24 and Figure 27. Comparing this with the results of Figure 23 and Figure 26, it is noted that there is a substantial improvement in segmentation, especially in the region of the aneurysm. But still, there are some false negative voxels near the singular point (near the middle of the aneurysm). This is because the level of intensity is very low, and the flow pattern is seriously corrupted by noise. A higher level of understanding of flow topology is required to tackle this problem, which is a subject of our current work.

\section{Conclusion}

We have derived a Maxwell-Gaussian mixture model for the background noise distribution of PC-MRA images generated by a phase-difference PC-MRA image post-processing algorithm. It has been shown that the Maxwell-Gaussian mixture 
model fits better than a Maxwell distribution for modelling background noise, which has been used in prior work [1]. Using this mixture model, we have proposed a statistical approach to aggregating speed and phase information available in PCMRA, and demonstrated that inclusion of phase information as a priori knowledge in the MRF model can improve the quality of segmentation, especially in the region within an aneurysm. Future work will include detection of the flow singular point, which may indicate the presence of an aneurysm, perhaps by using knowledge of flow topology [10] and through more detailed studies of application to a large number of aneurysms.

Acknowledgments: We thank Prof. J. Byrne and Dr. A. Martinez at Department of Radiology, Radcliffe Infirmary, Oxford for helpful discussions. A. Chung is funded by a postgraduate scholarship award from the Croucher Foundation, HK.

\section{References}

1. A.H. Andersen \& J.E. Kirsch, "Analysis of noise in phase contrast MR imaging", Med. Phy., 23(6), June 1996.

2. J. Besag, "On the Statistical Analysis of Dirty Pictures", J. Royal Statistical Society (B), 48(3):259-302, 1986.

3. C.M. Bishop, Neural Networks for Pattern Recognition, Clarendon Press, Oxford, 95.

4. M.E. Brummer, R.M. Mersereau, R.L. Eisner \& R.R.J. Lewine, "Automatic Detection of Brain Contours in MRI Data Sets", TMI 12(2):153-166, June, 1993.

5. A.C. Burleson, et. al., "Computer Modeling of Intracranial Saccular and Lateral Aneurysms for the Study of Their Hemodynamics", Neurosurgery, 37(4):774-784, 95.

6. A.C.S. Chung \& J.A. Noble, "Statistical 3D vessel segmentation using a Rician distribution", In MICCAI'99, pp.82-89 and MIUA'99, pp.77-80.

7. S. Geman and D. Geman, "Stochastic Relaxation, Gibbs Distributions, and the Bayesian Restoration of Images", PAMI, 6(6):721-741,1984.

8. Y.P. Gobin, J.L. Counord, P. Flaud \& J. Duffaux, "In Vitro study of haemodynamics in a giant saccular aneurysm model", Neuroradiology, 36:530-536, 1994.

9. K. Held, E.R. Kops, B.J. Krause, W.M. Wells, R. Kikinis \& H.W. Muller-Gartner, "Markov Random Field Segmentation of Brain MR Images", TMI, 16(6):878-886, 97.

10. J.L. Helman \& L. Hesselink, "Visualization of Vector Field Topology in Fluid Flows", IEEE Comp. Graphics and Appl., 11(3):36-46, 1991.

11. T. Kapur, W.E.L. Grimson, R. Kikinis \& W.M. Wells, "Enhanced Spatial Priors for Segmentation of Magnetic Resonance Imagery", MICCAI'98, pp.457-468.

12. K. Krissian, G. Malandain \& N. Ayache, "Model Based Detection of Tubular Structures in 3D Images", INRIA-Technical Report 3736, 1999.

13. B.P. Lathi, Modern Digital and Analog Communication Systems, Ch. 11, HaultSaunders Intl. Ed., 1983.

14. L.M. Lorigo, O. Faugeras, W.E.L Grimson, R. Keriven, R. Kikinis \& C.F. Westin, "Co-dimension 2 Geodesic Active Contours for MRA Segmentation", IPMI'99, pp.126-139.

15. T. McInerney \& D. Terzopoulos, "Medical Image Segmentation Using Topologically Adaptable Surface", CVRMed'97, pp.23-32.

16. N.J. Pelc, M.A. Bernstein et. al., "Encoding Strategies for Three-Direction PhaseContrast MR Imaging of Flow", JMRI, 1:405-413, 1991.

17. H.J. Steiger, et. al., "Computational Simulation of Turbulent Signal Loss in 2D Timeof-Flight Magnetic Resonance Angiograms", MRM, 37:609-614, 97. 
18. P. Summers, A.C.S. Chung \& J.A. Noble, "Impact of Image Processing Operations on MR Noise Distributions", In Proc. of $8^{\text {th }}$ ISMRM, 2000.

19. P. Summers, A.H. Bhalerao \& D.J. Hawkes, "Multiresolution, Model-Based Segmentation of MR Angiograms", JMRI, 7:950-957, 1997.

20. Y. Zhang, S. Smith \& M. Brady, "Segmentation of brain MR images using Markov random fields", $M I U A$ '99, p.65-68.

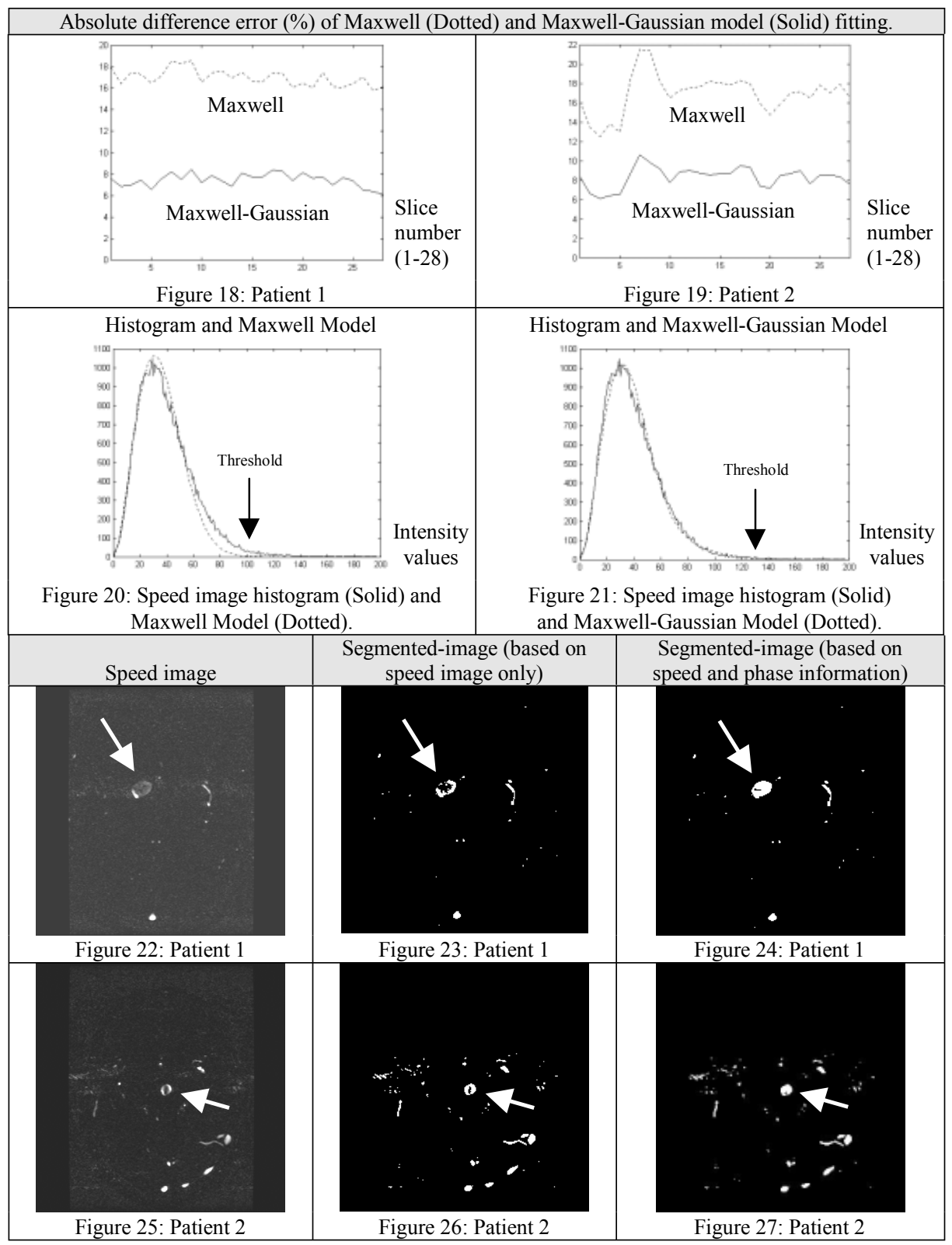

